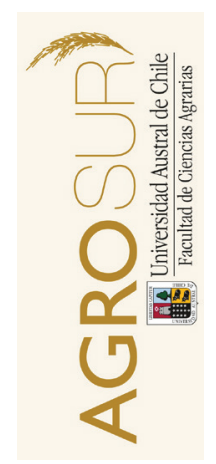

\title{
Efecto de la enmienda orgánica con lodos de cerdo sobre el establecimiento de Lolium perenne en relaves mineros
}

\author{
Effect of swine slurry as organic amendment of mine tailings on the \\ establishment of Lolium perenne
}

\author{
España, H. ${ }^{a}$, Quinteros, J. ${ }^{b}$, Ginocchio, R. ${ }^{b, c}$, Bas, F. ${ }^{a}$, Arias, R. ${ }^{d}$, Gandarillas, M. ${ }^{a, d *}$ \\ ${ }^{a}$ Departamento de Ciencias Animales, Pontificia Universidad Católica de Chile. \\ ${ }^{b}$ Departamento de Ecosistemas y Medio Ambiente, Pontificia Universidad Católica de Chile. \\ ${ }^{c}$ Center of Applied Ecology and Sustainability (CAPES), Pontificia Universidad Católica de Chile. \\ ${ }^{d}$ Instituto de Producción Animal, Universidad Austral de Chile.
}

\begin{tabular}{l} 
A R T I C L E I N F O \\
\hline Article history: \\
Received 29.06.2016 \\
Accepted 18.12.2016 \\
\hline Keywords: \\
Pig slurry \\
Lolium perenne \\
Organic amendment \\
Mine remediation \\
\hline Original Research Article, \\
Special Issue: Pastures for \\
Sustainable Productions Systems \\
\hline *Corresponding author: \\
Mónica Gandarillas \\
E-mail address: \\
monica.gandarillas@uach.cl
\end{tabular}

\begin{abstract}
A B S T R A C T
In Chile, pork production has generated significant income due to the large exportation volume. However, intensive pork production leads to an increase in the production of organic wastes, which may pose environmental problems. Chile is also the largest copper mine producer and the large-scale mines generate massive amounts of tailings which are stored in tailings storage facilities (TSFs). Under the new mine closure legislation, TSFs must be properly closed in order to minimize their environmental impact. This exploratory research was conducted to assess the efficacy for using pig sludge derived from pig slurry treatment plants as organic amendment for the remediation of TSFs. For this, mine tailings were amended with pig sludge at doses of 50, 100 and $200 \mathrm{t} \mathrm{ha}^{-1}$ plus two controls (agricultural soil and unamended tailings). One liter pots were filled with experimental substrates (replicated pots) and maintained in a greenhouse $\left(16^{\circ}-25^{\circ} \mathrm{C}\right)$ for a period of 15 days to allow substrate stabilization through irrigation. They were then seeded with $0.6 \mathrm{~g}$ of Lolium perenne (ryegrass) seeds (bioindicator plant), and maintained for a total of 8 weeks. Evaluated variables were aerial and root biomass (fresh and dry), percentage of roots cover, and plant height. Results showed that incorporation of pig sludge into tailings strongly inhibited seed germination, even at the lower dose used, thus resulting in significantly lower plant yield and growth when compared with both controls (agricultural soil and unamended tailings).
\end{abstract}

\section{RESUMEN}

En Chile, la carne de cerdo genera importantes ingresos debido al gran volumen de exportación. Sin embargo, la producción intensiva de cerdos genera grandes cantidades de desechos orgánicos, los que pueden conllevar problemas ambientales. Chile es además el primer productor de cobre del mundo y las minas de gran escala generan grandes volúmenes de relaves, los que son embancados en depósitos de relaves. Bajo las nuevas normativas de cierre de faenas mineras, estas estructuras deben ser cerradas adecuadamente para minimizar los impactos ambientales. La presente investigación exploratoria tuvo por objetivo evaluar la eficacia del uso de lodos de plantas de tratamiento de purines de cerdo como enmienda orgánica para la remediación de depósitos de relaves mineros. Relaves mineros fueron acondicionados con lodos de purines en dosis de 50, 100 y $200 \mathrm{t}$ ha $^{-1}$ más dos controles (suelo agrícola y relave sin enmendar). Macetas de $1 \mathrm{~L}$ se llenaron con los sustratos experimentales ( 4 repeticiones) y se mantuvieron en invernadero $\left(16^{\circ}-25^{\circ} \mathrm{C}\right.$ ) por 15 días para estabilizarlos con riego. Posteriormente se sembró con 0,6 g de semillas de Lolium perenne (planta bioindicadora) y se mantuvieron por 8 semanas. Las variables respuesta evaluadas fueron la biomasa aérea y radicular (fresca y seca), el porcentaje de cobertura radicular y la altura. Los resultados mostraron que la incorporación de lodos de purines de cerdo en los relaves inhibió fuertemente la germinación, aún a las dosis más bajas, resultando en productividad y crecimiento vegetal significativamente menores que los controles usados (suelo agrícola y relaves sin enmendar).

Palabras clave: lodo de cerdo, Lolium perenne, enmienda orgánica, remediación minera.

\section{INTRODUCCIÓN}

En Chile, la producción de carne de cerdo se ha incrementado desde 261 mil toneladas en el año 2000 a
524 mil toneladas en el año 2015 (Giacomozzi y Amunátegui, 2016). Detrás de este gran crecimiento han surgido numerosos desafíos tales como la mejora en la calidad e inocuidad en los alimentos, los planes de 
manejo para proteger la salud humana y animal y la gestión en los temas medio ambientales (Peralta et al., 2005). En nuestro país, el cerdo comercializado proviene mayoritariamente desde sistemas intensivos, el que si bien se caracteriza por ser altamente productivo, conlleva necesariamente a la preocupación por el manejo de purines debido a que una mala gestión podría terminar en la contaminación de cuerpos de agua, suelos y por las emisiones al aire de gases, polvo, ruido y olores (Espejo y García, 2009).

Por lo tanto, un desafío constante por parte de la industria porcina intensiva es la gestión y disposición adecuada de los residuos generados. En las plantas de tratamiento, el purín de cerdo -una mezcla de alto contenido de agua, orina y heces junto a restos de alimento y/o cama desperdiciado- es obtenido en los pabellones de producción y posteriormente recepcionado en estanques homogenizadores que almacenan y mezclan los contenidos. Posteriormente, el purín es separado en una fracción sólida denominada "guano" y en una fracción líquida mediante distintos procesos físicos. El guano puede utilizarse como abono para el suelo o bien, compostarse. La fracción líquida en cambio recibe distintos tipos de tratamiento (procesos de nitrificación y desnitrificación en estanques reactores) para facilitar su gestión y disposición. Cualquiera sea el sistema empleado, de esta fracción líquida, se obtiene el "lodo", que corresponde a las partículas finas que se consiguen por decantación luego de su tratamiento. En las plantas de tratamiento de purines, el lodo sigue siendo un residuo problemático, debido a la alta concentración de sales (conductividad eléctrica), metales (cobre y zinc) y nutrientes (sobre todo nitrógeno). Por ello, se hace indispensable su estabilización posterior (Peralta et al., 2005) para evitar convertirse en un problema de contaminación ambiental y generador de molestias por olores.

Por otra parte, en los últimos 150 años se han generado en Chile grandes volúmenes de desechos mineros sólidos producto de la explotación de gran escala de minerales de cobre dentro de la zona norte y centro del país (Ginocchio y León-Lobos, 2010). La disposición de estos volúmenes de residuos sólidos en el medio ambiente, tales como los depósitos de relaves, puede resultar en la generación de diversos riesgos ambientales, particularmente cuando su disposición, manejo y/o abandono son inadecuados. El abandono inadecuado determina que los relaves embancados sean dispersados al entorno (generación de polvo) o que reaccionen con el agua y el oxígeno que infiltra el material, produciendo drenaje ácido y lixiviación de metales al entorno (Ginocchio y León-Lobos, 2010). Las actuales disposiciones mineras, tal como la Ley de Cierre y Abandono de Faenas e Instalaciones Mineras (Ley 20.551 de 2011) establecen la obligatoriedad de formular y ejecutar planes de cierre para los depósitos de relaves post-operativos, de forma de asegurar la protección de la salud y seguridad de las personas y reducir el impacto ambiental de estas estructuras.

Estos depósitos constituyen una fuente potencial de contaminación para el área circundante debido a la alta posibilidad de movilización del material particulado a las zonas aledañas por procesos erosivos (eólicos y lluvias intensas), debido a los altos contenidos de metales y metaloides (ej. arsénico) (Conesa et al., 2007; Kabas et al., 2012). La dispersión de los relaves al entorno puede afectar negativamente a la salud humana de habitantes cercanos (Mendez y Maier, 2008), a los ecosistemas por la pérdida del hábitat y vegetación, la alteración de la calidad de las aguas y la acumulación de metales en las cadenas alimentarias (Kumar y Maiti, 2015), especialmente en zonas Mediterráneas semiáridas (Santibáñez et al., 2008). Así, existe necesidad de intervención humana para evitar la contaminación en áreas adyacentes (Ssenku et al., 2014). Una de las tecnologías usadas internacionalmente para asegurar la estabilidad física y química de los depósitos de relaves post-operativos es el establecimiento de una cubierta de vegetación ad-hoc sobre los relaves (Mendez y Maier, 2008; Kabas et al., 2014). Sin embargo, existen factores edafológicos propios de estos residuos que limitan su estabilización con cubiertas vegetales, como lo son: la alta compactación, la limitada capacidad de intercambio catiónico, la falta de materia orgánica, los bajos contenidos de carbono y macro nutrientes como nitrógeno y fósforo, los niveles extremos de $\mathrm{pH}$ (alcalino o ácido) y los altos contenidos de metales (Verdugo et al., 2011; Kabas et al., 2014, Ussiri y Lal, 2005). El uso de residuos orgánicos como enmiendas orgánicas ha demostrado ser efectivo para la remediación de residuos mineros masivos y suelos degradados químicamente por la minería (Cárcamo et al., 2012; Arellano y Ginocchio, 2013).

La aplicación de residuos orgánicos en relaves mejora la concentración de materia orgánica y nutrientes; a su vez disminuye la biodisponibilidad de metales (Bernal et al., 2007; Bernal et al., 2009), mejora la estructura del relave, incrementa la biomasa y diversidad bacteriana y fúngica (Farrel et al., 2015; De Varennes et al., 2010; De la Fuente et al., 2010; Clemente et al., 2012), y favorece la activación de los ciclos biogeoquímicos (Zanuzzi et al., 2009; Kabas et al., 2012; Zornoza et al., 2012). Al establecer coberturas vegetales se minimiza la erosión, la dispersión de contaminantes (Clemente et al., 2012; Ginocchio y León-Lobos, 2010) y mejora los valores estéticos (Vangronsveld y Cunningham, 1998). A pesar de esto, en Chile el uso integrado de residuos orgánicos con residuos mineros aún no ha sido considerado en las políticas públicas de gestión integral y racional de los residuos sólidos. Esta alternativa puede constituir una oportunidad para que los operadores mineros puedan dar cumplimiento a los nuevos requerimientos de remediación ambien- 
tal establecidos en la reciente Ley de Cierre de faenas e instalaciones mineras, y para que los generadores de residuos orgánicos -como las plantas de producción de cerdo- puedan valorizar sus residuos efectivamente como enmiendas orgánicas y nutricionales, dando una disposición final de estos que considere su valor como enmienda (Arellano y Ginocchio, 2013).

Generalmente, para la remediación de suelos mineros es recomendable utilizar especies nativas, que sean tolerantes a altas concentraciones de metales. Sin embargo, en etapas iniciales de remediación también se utilizan especies forrajeras por su rápido crecimiento y establecimiento de coberturas vegetales. Las principales especies de pasto tolerantes a metales son: Lolium perenne L., Festuca rubra L., Poa pratensis L., Agrostis capillaris L., Agrostis tenuis Sibth., Lolium italicum Lam. y Festuca arundinacea Schreb. (Kacprzak et al., 2014). La especie L. perenne empleada en el presente estudio, es una especie utilizada para la modelación de la absorción de metales en suelos contaminados (Hough et al., 2005), dado que es una metalófita facultativa ya que puede sobrevivir tanto en lugares metalíferos como en suelos normales (Smith y Bradshaw, 1979). Al acumular metales en sus raíces y evitar la translocación de estos metales a la parte aérea, es una especie modelo ampliamente utilizada como bioindicadora en programas experimentales de fitoestabilización (Bidar et al., 2007; Santibáñez et al., 2008; Cárcamo et al., 2012), ya que es posible detectar eficazmente los cambios producidos en los sustratos.

Recientemente, se ha observado un incremento en el uso de purines y compost de estiércol de cerdo como enmienda de suelos contaminados por la minería, especialmente en España (Martínez-Fernández et al., 2014; Pardo et al., 2014; Zanuzzi et al., 2013; Zornoza y Faz, 2013; Zornoza et al., 2013; Zornoza et al., 2012), donde al igual que en Chile existen depósitos de relaves mineros en zonas semiáridas. Cabe señalar que no existen estudios en los que se apliquen lodos de tratamiento de purín como enmienda de relaves mineros, como es el caso de lodos sanitarios (biosólidos) en los que se ha tenido éxito con el establecimiento de especies vegetales (Santibáñez et al., 2008; Verdugo et al., 2011). Por ello, es importante el estudio del uso de este residuo en relaves mineros. Por lo tanto, el objetivo de este estudio exploratorio fue evaluar a escala experimental, la eficacia del uso de los lodos de purines de cerdo como enmienda orgánica para la remediación de relaves mineros.

\section{MATERIALES Y MÉTODOS}

El presente estudio se basó en la metodología usada por Cárcamo et al. (2012), debido a la similitud que presentaba ese trabajo con los objetivos del presente estudio; es decir, el analizar los efectos de distintas dosis de una enmienda orgánica sobre un sustrato mineral.

\section{Relaves mineros y control positivo}

Se utilizó una muestra de relave minero proveniente del tranque abandonado Huana (30 42' $32^{\prime \prime}$ LS $70^{\circ}$ 57' 14" LW) ubicado en la Región de Coquimbo, provincia de Limarí, comuna de Monte Patria. Las muestras se obtuvieron a $20 \mathrm{~cm}$ de profundidad con un porcentaje de humedad de $0,41 \%$ y se almacenaron por un corto período de tiempo en bolsas plásticas selladas en los invernaderos de investigación de la Facultad de Agronomía e Ingeniería Forestal (FAIF) de la Pontificia Universidad Católica de Chile (UC). Sus características físico-químicas se detallan en la Tabla 1. Se utilizó este relave sin enmendar como control negativo (C-), mientras que se recolectó una muestra de $6 \mathrm{~kg}$ de suelo de uso agrícola proveniente de la unidad de Biohuerto de la Pontificia Universidad Católica de Chile para ser usado como control positivo $(\mathrm{C}+)$. El C+ fue recolectado con palas (0-20 $\mathrm{cm}$ de profundidad). Ambos controles fueron tamizados a $<2 \mathrm{~mm}$ y secados en estufa a $60^{\circ} \mathrm{C}$ por 12 horas.

\section{Lodos de purines}

Se colectaron 4 kilos de lodos después de la etapa de centrifugación de lodos primarios y secundarios del tanque de lodos en una planta de tratamiento de purines de cerdo de la zona central de Chile. Se denominan lodos, ya que se han obtenido desde la fracción líquida,

Tabla 1. Características fisicoquímicas de los relaves del tranque Huana.

Table 1. Physicochemical characteristics of tailings from Huana tailing dump.

\begin{tabular}{lc}
\hline Parámetros & Valor \\
\hline $\mathrm{pH}$ & 7,8 \\
Conductividad Eléctrica $\left(\mathrm{ds} \mathrm{m}^{-2}\right)$ & 2,0 \\
Capacidad de Intercambio Catiónico $_{\left(\mathrm{cmol}_{(+)} \mathrm{kg}^{-1}\right)}$ & 3,77 \\
Carbono Orgánico (\%) & 0,03 \\
Cu total $\left(\mathrm{mg} \mathrm{L}^{-1}\right)$ & 1.094 \\
Zn total $\left(\mathrm{mg} \mathrm{L}^{-1}\right)$ & 108 \\
Fe total $\left(\mathrm{mg} \mathrm{L}^{-1}\right)$ & 49.939 \\
Ca total $\left(\mathrm{mg} \mathrm{L}^{-1}\right)$ & 8.390 \\
As total $\left(\mathrm{mg} \mathrm{L}^{-1}\right)$ & 4 \\
Textura & \\
Fracción $<2 \mu \mathrm{m}(\%)$ & 5 \\
Fracción entre 2 y $50 \mu \mathrm{m}(\%)$ & 14 \\
Fracción entre 50 y $2000 \mu \mathrm{m}(\%)$ & 80 \\
\hline
\end{tabular}


que a su vez fue la resultante de una primera separación desde la fase sólida del purín crudo mediante un método físico (prensa tornillo). El lodo primario fue separado del líquido mediante el uso de polímeros y luego del paso por el tratamiento de lagunas aeróbicas y anaeróbicas donde se obtuvo el lodo secundario (caracterización de la mezcla de lodos en Tabla 2).

\section{Sustratos experimentales}

Se llevó a cabo un ensayo bajo condiciones controladas de invernadero $\left(16-25^{\circ} \mathrm{C}\right)$, donde se utilizaron los lodos de tratamiento de purín en dosis incrementales de 50, 100 y 200 t ha $^{-1}$ de materia seca como enmienda orgánica del relave minero, T1, T2 y T3, correspondientemente. Las dosis usadas se basan en la normativa vigente de aplicación de lodos sanitarios a suelos agrícolas en Chile (50 y $100 \mathrm{t} \mathrm{ha}^{-1}$ ), y una tercera correspondiente al doble del valor máximo permitido (200 t ha ${ }^{1}$ ), ya que la aplicación de lodos en relaves mineros no está normada en Chile (Ministerio de Medio Ambiente, 2010) y las líneas base de materia orgánica y Nitrógeno de los relaves es prácticamente cero. Previamente, se secó el lodo en estufa a $60{ }^{\circ} \mathrm{C}$ hasta peso constante y se determinó su porcentaje de materia seca para realizar los cálculos para la dosificación de los lodos en base húmeda. Los sustratos experimentales para cada tratamiento de este ensayo se prepararon mezclando $2 \mathrm{~kg}$ de relaves en contenedores plásticos de $5 \mathrm{~L}$; se añadió las tres dosis incrementales correspondientes a cada tratamiento de lodo de tratamiento de purín. Las mezclas se hicieron girar sobre un roller automatizado (Tecco YGR) a 11 revoluciones por minuto, durante 20 minutos (Cárcamo et al., 2012) y posteriormente se verificó la homogenización de forma manual. Luego de esto, el sustrato obtenido de cada tratamiento, el C+y el

Tabla 2. Características de los lodos utilizados como enmienda orgánica.

Table 2. Characteristics of the pig sludges used as organic amendment.

\begin{tabular}{lc}
\hline Parámetros & Valor \\
\hline $\mathrm{pH}$ & 6,20 \\
Conductividad Eléctrica $\left(\mathrm{ds} \mathrm{m}^{-2}\right)$ & 10,15 \\
Materia Orgánica (\%) & 71,25 \\
Carbono Orgánico (\%) & 39,58 \\
Nitrógeno (\%) & 5,49 \\
Relación C/N & 9,08 \\
Cu total $\left(\mathrm{mg} \mathrm{kg}^{-1}\right)$ & 1.968 \\
Zn total (mg kg-1) & 3.519 \\
Humedad (\%) & 78,75 \\
\hline
\end{tabular}

C- fueron distribuidos en vasos plásticos de 1 litro, considerando cuatro réplicas por tratamiento (total de 20 vasos), con drenaje en la base; se llenó cada recipiente hasta completar 800 gramos del sustrato.

\section{Capacidad de Campo}

Se determinó a todos los sustratos experimentales (C-, C+, T1 a T3) la capacidad de campo mediante el método ISO 11269-1:1993(E) (ISO, 1993). Para esto, se utilizaron tubetes plásticos previamente pesados y se taparon en su parte inferior con algodón sintético, también previamente pesado. Los sustratos experimentales se secaron a $45{ }^{\circ} \mathrm{C}$ hasta peso constante, posteriormente se rellenaron los tubetes con los sustratos de cada tratamiento y controles, con tres réplicas cada uno. Estos fueron dispuestos en una gradilla y sumergidos dentro de un recipiente con agua desioniza$\mathrm{da}$, permitiendo que el agua ascendiera por el sustrato por capilaridad hasta que la superficie de cada sustrato estuviese saturada, momento en el cual se removió el recipiente con agua de manera de permitir el drenaje por gravedad, y se cubrió la parte superior de los tubetes con plástico, para evitar la evaporación. Los tubetes se dejaron drenar por un periodo de 12 horas para posteriormente remover el algodón y volver a pesar los tubetes, esta vez con los sustratos a $100 \%$ de capacidad de campo. Se consideró la diferencia del peso entre el tubete al $100 \%$ de capacidad de campo con el peso del tubete con el sustrato seco como la cantidad de agua que tiene el sustrato cuando se encuentra a $100 \%$ de capacidad de campo, y se utilizó este dato para determinar el volumen de riego del $70 \%$ de capacidad de campo para cada recipiente.

\section{Montaje y Desarrollo}

Los recipientes experimentales se mantuvieron en condiciones controladas en un invernadero de la FAIF, durante 8 semanas. La temperatura fue controlada $\left(16\right.$ a $\left.25^{\circ} \mathrm{C}\right)$, pero no el fotoperiodo ni la humedad relativa. Durante las dos primeras semanas (fase de tiempo para la estabilización de los sustratos), se controló día por medio la humedad de los sustratos experimentales, pesando los recipientes con sustrato con una balanza de $1 \mathrm{~g}$ de precisión y reponiendo con agua de la llave el volumen faltante de agua para alcanzar el $70 \%$ de capacidad de campo. Al inicio de la tercera semana, de un total de ocho, todos los recipientes fueron sembrados con 0,6 gramos de Lolium perenne (ballica inglesa) variedades Apple, Saint y Stellar y cubiertos con una capa de 1 $\mathrm{cm}$ aproximadamente del mismo sustrato experimental. Se mantuvo el riego a 70\% de capacidad de campo, controlado día por medio. La posición de los recipientes fue alterada aleatoriamente cada vez que se revisó la humedad del suelo, para evitar efectos de borde. 


\section{Variables determinadas}

Al cabo de las ocho semanas se finalizó el ensayo experimental. Previa a la cosecha, se midió la altura alcanzada por las plantas de Lolium perenne en cada tratamiento experimental usando una regla metálica (5 mediciones al azar por recipiente). Posteriormente, se cosechó la biomasa aérea de cada recipiente a ras de suelo, lavando los tallos con agua, secando el exceso de agua con papel absorbente y masando la biomasa fresca (peso fresco). La biomasa fue secada a $45^{\circ} \mathrm{C}$ hasta alcanzar peso constante ( 48 horas), y luego fue pesada nuevamente, registrando el peso seco. Los recipientes fueron luego cortados longitudinalmente para registrar la cobertura de raíces. La cobertura radicular (en porcentaje) fue determinada mediante el uso de una grilla de $1 \mathrm{x} 1 \mathrm{~cm}$ sobrepuesta al sustrato expuesto de una de las mitades de los recipientes, registrándose el número de intersecciones de la grilla que entró en contacto con las raíces. El porcentaje de cobertura fue calculado considerando la frecuencia de los contactos con el número de intersecciones totales registradas. Para registrar la biomasa radicular se seleccionaron 3 recipientes al azar por cada tratamiento y se separaron manualmente las raíces del sustrato y posteriormente con la ayuda de un tamiz. Las raíces fueron luego lavadas con agua, secadas a $45^{\circ} \mathrm{C}$ por 48 horas y pesadas para registrar su peso seco. El sustrato separado de las raíces fue tamizado nuevamente, secado a $60^{\circ} \mathrm{C}$, guardado en bolsas zip-lock y enviado a los laboratorios de Análisis de Suelos y Foliar de la UC, junto con una muestra del sustrato originales que no fueron utilizados con el fin de realizar análisis de $\mathrm{pH}$ y conductividad eléctrica (caracterización de sustratos en Tabla 3).

\section{Diseño experimental y análisis estadístico}

El modelo experimental utilizado correspondió a un diseño completamente al azar con tres tratamien- tos y dos testigos (positivo y negativo). El número de unidades experimentales y observacionales para biomasa aérea y radical fue de 4 por tratamiento. En tanto, para la variable altura de la planta el número de unidades observacionales fue de 5 por réplica. Se realizaron pruebas de normalidad (Shapiro-Wilk) y homocedasticidad (Levene). El contraste de hipótesis se realizó mediante ANOVA de una vía y la prueba de comparación múltiple fue mediante test de Tukey. En los casos en que no se cumplió con los supuestos de homocedasticidad se utilizó la prueba de Wilcoxon y Kruskall-Wallis y la prueba de comparación múltiple de Wilcoxon. El nivel de significancia en todos los casos fue de 5\%. El modelo estadístico evaluado fue: $\mathrm{Y}_{\mathrm{ij}}=\mu$ $+\alpha_{\mathrm{i}}+\varepsilon_{\mathrm{ij},}$ Donde: $\mu$ corresponde al promedio de la población; $\alpha$ representa el efecto del "jvo" tratamiento y $\varepsilon$ es el error experimental asociado al iavo recipiente del jvo tratamiento. Finalmente, se estimaron regresiones lineales polinomiales de segundo grado para predecir la producción de biomasa aérea (MV y MS) en función de las toneladas de lodos de purines de cerdo aplicados al relave minero. Se utilizó el software estadístico JMP versión 11.0 SAS Institute, Carolina del Norte, Estados Unidos.

\section{RESULTADOS}

La Tabla 4 presenta las medias robustas de la producción de biomasa aérea fresca y seca de las plantas de Lolium perenne al cabo de 8 semanas post siembra, con sus respectivos valores de error estándar de la media. Cabe señalar que ambas variables no fueron homocedásticas (test de Levene $\mathrm{P}=0,0028$ y $\mathrm{P}<0,0001$ para biomasa fresca y seca, respectivamente), razón por la cual ambas fueron analizadas mediante el test de Wilcoxon y Kruskal-Wallis. Hubo diferencias significativas entre los tratamientos evaluados tanto para la biomasa aérea producida, ya sea expresada en términos de biomasa fresca $(P=0,0018)$ como de biomasa seca

Tabla 3. Promedio de resultado de análisis de laboratorio de los sustratos posteriores a la cosecha.

Table 3. Results from analytical determinations of substrates after plant harvest.

\begin{tabular}{lcccccc}
\hline \multicolumn{1}{c}{ Análisis } & Unidad & C+ & C- & T1 & T2 & T3 \\
\hline pH susp & - & 7,51 & 7,90 & 7,08 & 7,16 & 7,03 \\
CE susp & $\mathrm{mS} \mathrm{cm}^{-1}$ & 1,37 & 1,00 & 1,40 & 1,53 & 1,9 \\
M.O. & $\%$ & 3,71 & 0,37 & 0,51 & 0,83 & 1,48 \\
N disp & $\mathrm{mg} \mathrm{kg}^{-1}$ & 136 & 28 & 185 & 184 & 252 \\
P Olsen & $\mathrm{mg} \mathrm{kg}^{-1}$ & 41 & 1,00 & 78 & 270 & 534 \\
K disp & $\mathrm{mg} \mathrm{kg}^{-1}$ & 192 & 57 & 185 & 307 & 489 \\
Cu total & $\mathrm{mg} \mathrm{kg}^{-1}$ & 93 & 1315 & 1325 & 1273 & 1123 \\
Cu ext & $\mathrm{mg} \mathrm{kg}^{-1}$ & 0,10 & $<0,02$ & 1,50 & 4,80 & 10,76 \\
\hline
\end{tabular}


Tabla 4. Medias y error estándar de la media (entre paréntesis) de la biomasa aérea (fresca y seca) para los tratamientos y controles.

Table 4. Mean and standard error (in brackets) of aerial biomass (fresh and dry) of treatments and controls.

\begin{tabular}{|c|c|c|c|c|c|}
\hline Tratamiento & \multicolumn{2}{|c|}{$\begin{array}{l}\text { Biomasa fresca, } \\
\text { gramos }^{*}\end{array}$} & \multicolumn{3}{|c|}{$\begin{array}{l}\text { Biomasa seca, } \\
\text { gramos }^{* *}\end{array}$} \\
\hline $\mathrm{C}+$ & 2,601 & $(0,50) \mathrm{a}$ & 1,050 & $(0,225)$ & $\mathrm{a}$ \\
\hline $\mathrm{C}-$ & 1,550 & $(0,06) \mathrm{b}$ & 0,550 & $(0,025)$ & $\mathrm{a}$ \\
\hline $\mathrm{T} 1$ & 1,275 & $(0,28) a b$ & 0,495 & $(0,046)$ & $\mathrm{a}$ \\
\hline $\mathrm{T} 2$ & 0,178 & $(0,03) \mathrm{c}$ & 0,050 & $(0,007)$ & $\mathrm{b}$ \\
\hline T3 & 0,025 & $(0,01) d$ & 0,018 & $(0,007)$ & $\mathrm{b}$ \\
\hline
\end{tabular}

${ }^{*} \mathrm{P}=0,0018$ test de Wilcoxon y Kruskal-Wallis

${ }^{* *} \mathrm{P}=0,0027$ test de Wilcoxon y Kruskal-Wallis

Letras distintas dentro de cada columna indican diferencias significativas entre tratamientos según test de Wilcoxon.

$\mathrm{C}+$, suelo; C-, relaves; T1, relaves con $50 \mathrm{t} \mathrm{ha}^{-1}$ (materia seca) lodos purines; T2, relaves con $100 \mathrm{t} \mathrm{ha}^{-1}$ (materia seca) lodos purines; $\mathrm{T} 3$, relaves con $200 \mathrm{t} \mathrm{ha}^{-1}$ (materia seca) lodos purines.
$(P=0,0027)$, lográndose en el primer caso mejores rendimientos en el control positivo $(\mathrm{C}+$; suelo agrícola) y T1, pero sin diferencias entre $\mathrm{T} 1 \mathrm{y}$ el control negativo (C-; relaves). En la medida que se aumentó la incorporación de lodos el rendimiento disminuyó drásticamente, presentando T3 el rendimiento más bajo $(1,0 \%$ de la producción de $\mathrm{C}+\mathrm{y} 1,6 \%$ respecto de $\mathrm{C}$-). Por otra parte, al expresar la biomasa aérea producida en términos de materia seca, sólo los tratamientos con mayores niveles de incorporación de lodos (T2 y T3) fueron inferiores a los testigos, alcanzado un 1,7\% y 3,2\% de lo producido en $\mathrm{C}+\mathrm{y} \mathrm{C}$-, respectivamente.

El efecto del lodo sobre la altura de plantas de $L O$ lium perenne se observa en la Figura 1. No se observa diferencias significativas entre T1 y C-, pero ambos redujeron su altura en prácticamente un $50 \%$ respecto de C+. De manera análoga se observa que la altura de las plantas fue disminuyendo en la medida que se incrementó el uso de lodos de purín de cerdo (T2 y T3). Así, las alturas de las plantas de T2 y T3 alcanzaron sólo un $31,0 \%$ y $16,1 \%$ de la altura alcanzada en $\mathrm{C}+\mathrm{y}$ prácticamente un $50 \%$ de la altura alcanzada en C-.

La Figura 2 (A y B) presenta las ecuaciones de predicción de biomasa aérea en función de la incorporación de lodos de purines de cerdo al relave minero. En la Fig. 2A se muestra la producción de biomasa aérea fresca cuyo mejor ajuste se logró con una ecuación cuadrática $\left(Y=3,05333-0,042375 x+0,0001362 x^{2}\right.$; $\mathrm{P}=0,0184$ ) la que explica un $74 \%$ de la variabilidad observada. En tanto en la Fig. 2B se presenta la ecua-

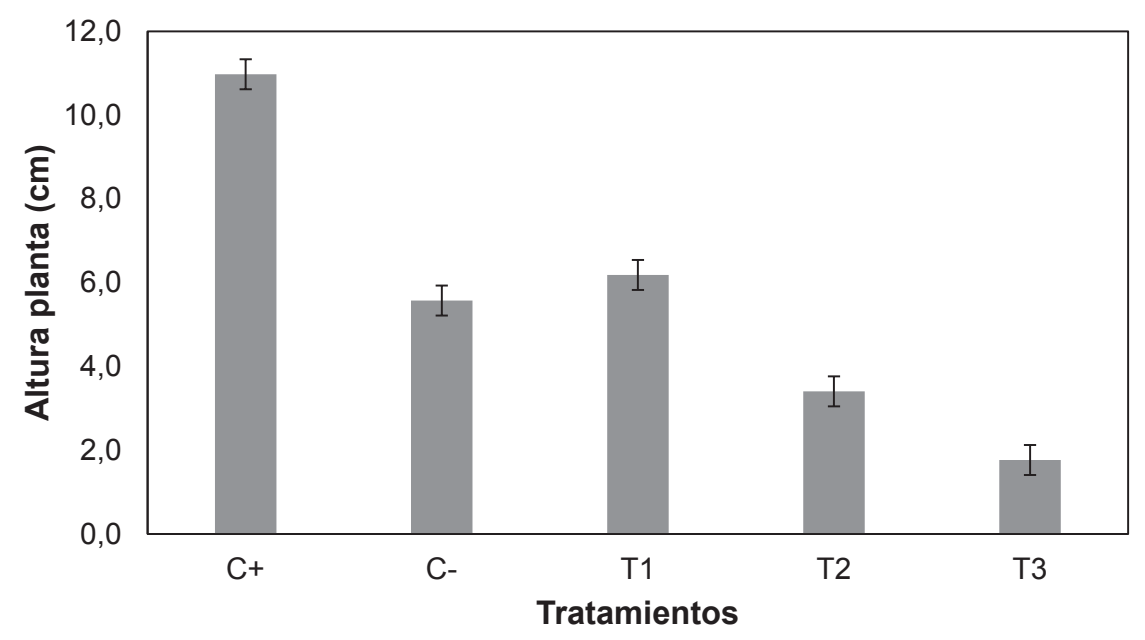

Figura 1. Medias de cuadrados mínimos de altura de plantas de Lolium perenne y error estándar de la media (entre paréntesis) establecida en distintos sustratos experimentales a 8 semanas post siembra. Letras distintas indican diferencias significativas entre tratamientos según test de Tukey. C+, suelo; C-, relaves; T1, relaves con 50 t ha-1 (materia seca) lodos; T2, relaves con 100 t ha $^{-1}$ (materia seca) lodos; T3, relaves con 200 t ha $^{-1}$ (materia seca) lodos.

Figure 1. Minimum square means and standard errors (in brackets) of plant height from individuals established on experimental substrates, 8 weeks after sowing. Different letters indicate significant differences among treatments according to Tukey test. C+, soil; C-, tailings; T1, tailings with $50 \mathrm{t} \mathrm{ha}^{-1}$ of sludges (dry weight); T2, tailings with $100 \mathrm{t} \mathrm{ha}^{-1}$ of sludges (dry weight); T3, tailings with $200 \mathrm{tha}^{-1}$ of sludges (dry weight). 
ción de predicción de la biomasa aérea seca, cuyo mejor ajuste también se logró con una ecuación cuadrática $\left(Y=1,22583-0,017475 x+0,00000572 x^{2} ; P<0,0001\right)$, pero con una mejor explicación de la variabilidad observada $\left(r^{2}=0,94\right)$.
La Figura 3 presenta los porcentajes de cobertura de raíces y la biomasa radicular (masa seca) producida bajo tres condiciones experimentales: $\mathrm{C}+, \mathrm{C}-\mathrm{y} \mathrm{T} 1$, dado que no se registraron valores en los otros tratamientos. En términos de cobertura, se observaron dife-

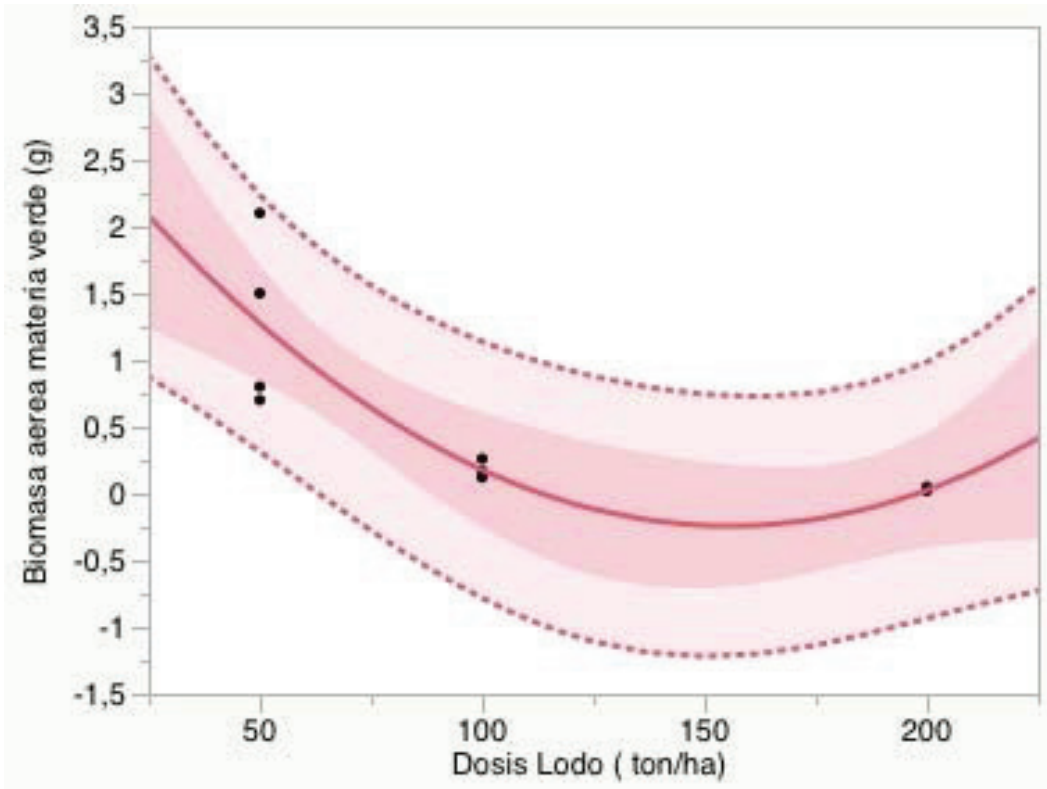

A

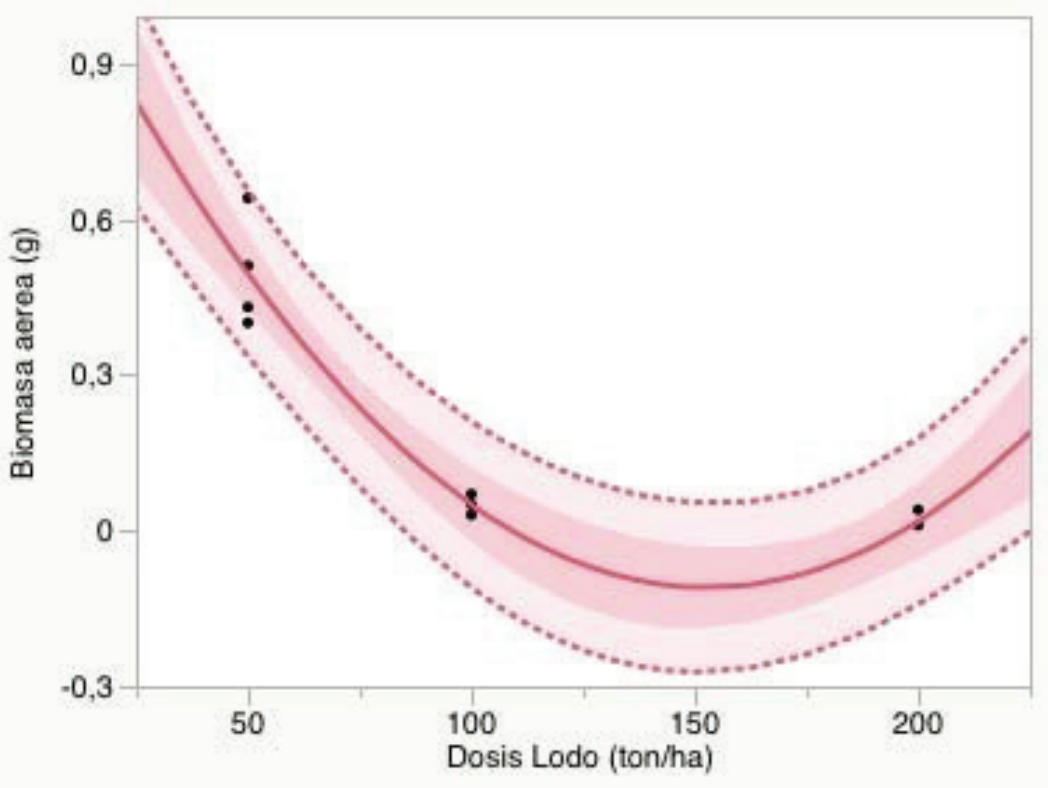

Figura 2. Ecuaciones de predicción de producción de biomasa aérea en función de lodos de purines de cerdo aplicados a un relave minero. A) Producción de biomasa aérea fresca (gramos) : $Y=3,05333-0,042375 x+0,0001362 x^{2} ; P=0,0184$ y r $r^{2}=$ 0,74. B) Producción de biomasa aérea seca (gramos): $Y=1,22583-0,017475 \mathrm{x}+0,00000572 \mathrm{x}^{2} ; \mathrm{P}<0,0001$ y r $\mathrm{r}^{2}=0,94$.

Figure 2. Predictive equations for aerial biomass production according to dose of pig sludge incorporated into mine tailings. A) Fresh aerial biomass (grams): $\mathrm{Y}=3.05333-0.042375 \mathrm{x} ; \mathrm{P}=0.0184$ and $\mathrm{r}^{2}=0.74$. B) Dry aerial biomass (grams): $\mathrm{Y}=1.22583$ $-0.017475 \mathrm{x}+0.00000572 \mathrm{x}^{2} ; \mathrm{P}<0.0001$ and $\mathrm{r}^{2}=0.94$ 


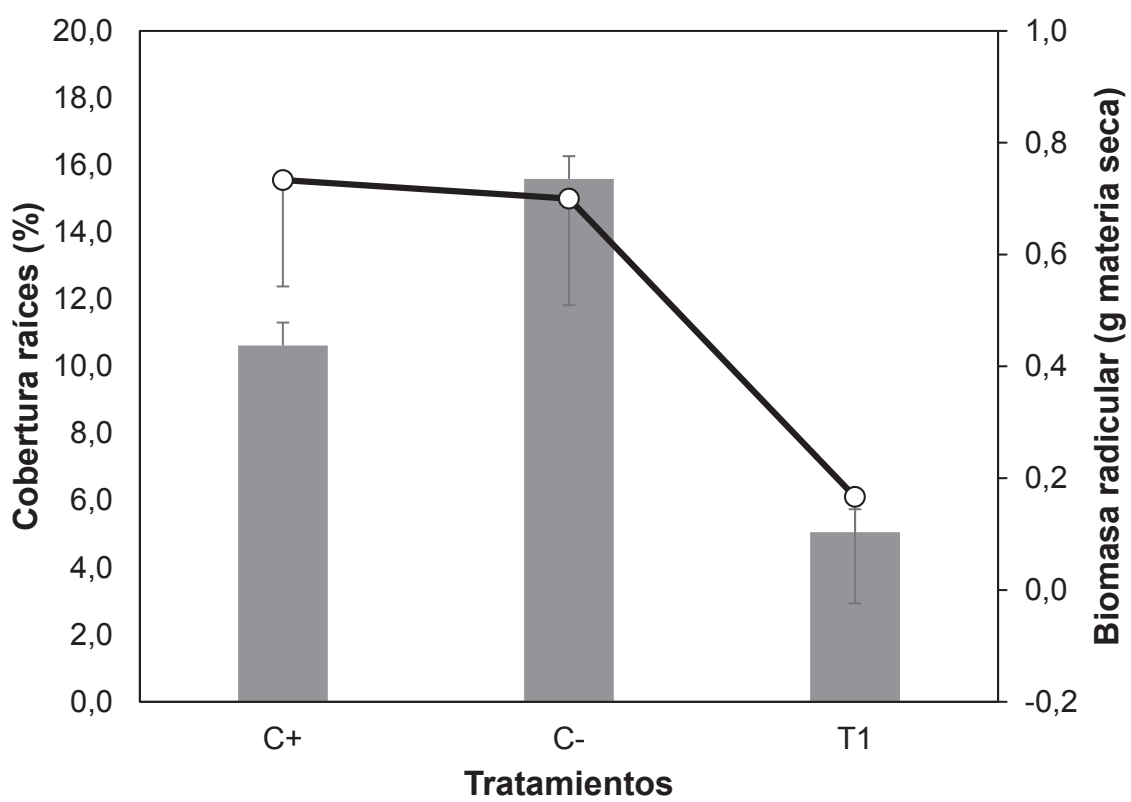

Figura 3. Medias de cuadrados mínimos de cobertura de raíces (barras) y media robusta de biomasa radicular (línea) de Lolium perenne establecida en tres condiciones experimentales (EEM). C+, suelo; C-, relaves; T1, relaves con 50 t ha ${ }^{-1}$ (materia seca) lodos purines.

Figure 3. Minimum square means of root cover (bars) and means of root biomass (line) of ryegrass under three experimental conditions. $\mathrm{C}+$, soil; C-, tailings; $\mathrm{T} 1$, tailings with $50 \mathrm{t} \mathrm{ha} \mathrm{a}^{-1}$ of pig sludges (dry weight).

rencias significativas entre los tratamientos evaluados ( $\mathrm{P}<0,0001)$, observándose la mayor cobertura en Cseguido por $\mathrm{C}+\mathrm{y}$ finalmente T1. En tanto, para el caso de biomasa radicular se observó una tendencia a menor producción de biomasa radicular en $\mathrm{T} 1$ respecto de los controles $(\mathrm{P}=0,0594)$.

\section{DISCUSIÓN}

Las diferencias observadas en productividad de L. perenne (biomasa aérea, altura de los vástagos y cobertura radicular) del tratamiento $\mathrm{C}$ - (relaves mineros) respecto del tratamiento $\mathrm{C}+$ (suelo agrícola) eran esperables. La carencia de macro nutrientes en los relaves no permite sustentar una productividad vegetal adecuada (Verdugo et al., 2011; Kabas et al., 2012, Zornoza y Faz, 2013). Sin embargo, la aplicación de dosis incrementales de lodos secundarios de purines de cerdo a los relaves no mejoró la productividad de L. perenne, siendo incluso menor a la obtenida en los relaves no enmendados (C-).

La incorporación de residuos orgánicos derivados de la producción porcina, a los relaves mineros aumenta los contenidos de nutrientes (N, P, K), materia orgánica y mejora los parámetros físicos y biológicos limitantes. Estas condiciones promueven el establecimiento y desarrollo vegetal (Zanuzzi et al., 2009 y Zornoza et al., 2013). No obstante, en nuestro estudio, aunque en los tratamientos T1 a T3 se encontraron niveles mayores de macronutrientes y de materia orgánica en el sustrato en relación a C- (Tabla 3); la productividad de L. perenne disminuyó.

Estos resultados se contraponen a otros estudios (Kabas et al., 2012; Zornoza et al., 2012; Pardo et al., 2011; Zanuzzi et al., 2009; Zanuzzi et al., 2013). Sin embargo, la diferencia pudo deberse a que en estos últimos se utilizaron purines de cerdo, estiércol de cerdo y compost de estiércol de cerdo, como enmiendas orgánicas de relaves mineros, y no lodos secundarios.

Respecto a las dosis utilizadas, existen estudios que utilizaron dosis superiores a la nuestra, ya sea de lodos compostados de cerdo (Martínez-Fernández et al., 2014) o de lodos sanitarios (biosólidos) como enmiendas orgánicas de relaves mineros (Verdugo et al., 2011; Santibáñez et al., 2011), y donde se obtuvieron productividades vegetales superiores. La reducción de la productividad de L. perenne (biomasa aérea, altura del vástago y cobertura radicular) en forma proporcional al incremento en la dosis de lodos secundarios de purines de cerdo aplicados a los relaves sugiere fitotoxicidad del sustrato (Zucconi et al., 1981). Por otra parte, Ye et al. (1999), reportaron una disminución de la biomasa radicular al incrementar las dosis de residuos porcinos, explicando que esto podría deberse a que existirían cantidades suficientes de nutrientes en el sustrato y que las plantas destinarían la mayoría de 
los recursos al desarrollo de biomasa aérea, lo que no sería el caso en el presente estudio. El escaso desarrollo radicular para T1 y la ausencia de desarrollo radicular en T2 y T3 (Figura 3), también podría atribuirse a fitotoxicidad del sustrato, ya que la germinación y el crecimiento radicular se redujeron notoriamente $(>50 \%)$ con la incorporación de los lodos secundarios y en una forma dosis-dependiente. En cuanto a la toxicidad de los lodos de cerdo, Tigini et al. (2016), reportan que los lodos de cerdo incluso compostados son ecotóxicos y conllevan un riesgo de contaminación ambiental. Sin embargo, esto se contradice con lo reportado por Alvarenga et al. (2015) quienes encontraron que los lodos de cerdo compostados poseen una muy alta estabilidad y un índice normal de germinación.

Existen varias causas posibles de fitotoxicidad atribuibles a los lodos secundarios de purines de cerdo. Una de ellas se debería a que la aplicación de residuos orgánicos inestables a este tipo de sustratos resulta en la generación de amoníaco y óxido de etileno, los que podrían causar la inhibición de la germinación y del desarrollo temprano de las raíces (Alvarenga et al., 2015). Para este caso en particular, sería interesante estudiar el efecto de tiempos de estabilización mayores a los utilizados en el presente estudio. Otra posible causa de fitotoxicidad podría ser la presencia de poliacrilamida catiónica (C-PAM) en los lodos secundarios de purín de cerdo. En este sentido, se ha atribuido toxicidad de los C-PAM para organismos de aguas superficiales (McLaughlin y Bartholomew, 2006), pero su fitotoxicidad requiere ser evaluada. Este compuesto se añade en altas dosificaciones en las plantas de tratamiento de purines, para separar los sólidos solubles de la fracción líquida del purín (Hjorth et al., 2010). Este tipo de compuestos floculantes son muy utilizados en los procesos de tratamiento secundario de purines, por ser más eficientes en la separación de los sólidos contenidos en aguas residuales (Vanotti y Hunt, 1999). Cabe señalar que la planta de tratamiento de purines de cerdo desde la que se obtuvieron los lodos secundarios usados en el presente estudio utiliza dicho compuesto.

Los residuos orgánicos de la producción intensiva de porcinos poseen altas concentraciones de $\mathrm{Cu}$ y $\mathrm{Zn}$, que derivan de los aditivos nutricionales suministrados a los cerdos (Popovic y Jensen, 2012; Lu et al., 2015). Adicionalmente, los relaves mineros derivados del procesamiento de minerales sulfurados de $\mathrm{Cu}$, como los usados en este estudio (Tabla 1), también poseen contenidos elevados de $\mathrm{Cu}$ y Zn (Verdugo et al., 2011). De esta forma, los altos contenidos de $\mathrm{Cu}$ y $\mathrm{Zn}$ que pudieran generarse en los relaves enmendados con lodos secundarios de purines podrían resultar en fitotoxicidad por metales (Adriano, 2001). Sin embargo, los resultados exitosos de otros estudios similares que han utilizado residuos porcinos con altos contenidos de $\mathrm{Cu}$ y $\mathrm{Zn}$ como enmiendas de relaves mineros, y la baja con- centración de $\mathrm{Cu}$ extractable encontrado (Tabla 3) en este estudio, sugieren que posiblemente estos residuos no serían fitotóxicos por metales (Pardo et al., 2011; Zornoza et al., 2012; Zanuzzi et al., 2013; Zornoza et al., 2013).

No es posible aseverar que únicamente existió fitotoxicidad en este ensayo, la que podría haber limitado la emergencia temprana $\mathrm{y} / \mathrm{o}$ el crecimiento posterior de las plantas (Hooper y Anderson, 2009). También podrían existir otros factores responsables de cambios en los relaves enmendados con lodos secundarios de purín de cerdo, tales como la textura y el grado de aireación del sustrato, entre otros. La incorporación de lodos secundarios de purín a sustratos de textura fina, como los relaves usados en el presente estudio, podría resultar en compactación y consecuentemente, en condiciones limitantes para el crecimiento de las plantas (Alcañiz et al., 2009). Así, sería adecuado evaluar el efecto de los lodos secundarios de purín sobre la estructura física de los relaves, de forma de asegurar una estabilización hidrogeoquímica eficiente de estos residuos mineros (Li y Huang, 2015).

Finalmente, se podrían descartar los contaminantes orgánicos potencialmente presentes en los lodos secundarios de purín, ya que de acuerdo con Alcañiz et al. (2009), muchos de ellos se degradan al ser incorporados al sustrato y no se les puede atribuir efectos directos fitotóxicos. También se descarta que la salinidad y/o el pH pudieran haber inhibido la germinación de las semillas, ya que estos parámetros no fueron afectados por la adición de las dosis más altas de lodos (Tabla 3). En el caso del pH del sustrato, ésta característica mejoró con la adición de los lodos secundarios de purines de cerdo, debido al efecto buffer de la materia orgánica de 7,9 en C- a 7,0 en T3.

Las distintas explicaciones propuestas para los resultados encontrados en el presente estudio deben ser, sin embargo, estudiadas con mayor profundidad en estudios con macetas más grandes de modo de representar mejor el comportamiento de la relación planta - suelo, y además que involucren mayor tiempo de crecimiento de las plantas y raíces.

\section{CONCLUSIONES}

Los lodos secundarios de purín de cerdo, en las dosis y condiciones usadas en este estudio (superiores a $\left.50 \mathrm{t} \mathrm{ha}^{-1}\right)$, aunque aumentan el contenido de nutrientes en el sustrato serían inadecuados como enmiendas de relaves mineros para el cultivo de L. perenne.

La productividad de L. perenne fue afectada negativamente y en forma dosis-dependiente por la incorporación de lodos secundarios de purines de cerdo a relaves mineros, siendo inhibida la germinación y el desarrollo radicular temprano, particularmente en las dosis más altas usadas (T2 y T3). 
Los resultados negativos obtenidos en este estudio podrían deberse a problemas de fitotoxicidad y/o a cambios en la textura del sustrato (compactación), generados por la incorporación de los lodos secundarios de purín a los relaves mineros. Las posibles fuentes de fitotoxicidad serían diversas y, es necesario realizar evaluaciones que consideren parámetros tales como el tiempo de estabilización del sustrato enmendado antes de la siembra y la fitotoxicidad del C-PAM contenido en los lodos secundarios de purín de cerdo, entre otros factores.

\section{AGRADECIMIENTOS}

Este artículo se encuentra dentro del proyecto denominado "Evaluación de alternativas de gestión de lodos de cerdo para su valorización como enmienda orgánica para la rehabilitación ecológica de relaves mineros", financiado por la Dirección de Investigación y Postgrado de la Facultad de Agronomía e Ingeniería Forestal de la Pontificia Universidad Católica de Chile. Los autores también agradecen el financiamiento del proyecto FB-0002-2014 de CONICYT

\section{REFERENCIAS}

Adriano, D.C., 2001. Trace elements in terrestrial environments. Biogeochemistry, bioavailability and risk of metals. Second edition. Springer. New York. doi: 10.1007/978-0-387-21510-5.

Alcañiz, J., Ortiz, O., Carabassa, V., 2009. Utilización de lodos de depuradora en restauración. Generalitat de Catalunya. http://www.ambiente.gov.ar/archivos/web/Probarros/file/3.pdf (acceso 13.06.2016).

Alvarenga, P., Mourinha, C., Farto, M., Santos, T., Palma, P., Sengo, J., Morais, M.-C., Cunha-Queda, C., 2015. Sewage sludge, compost and other representative organic wastes as agricultural soil amendments: Benefits versus limiting factors. Waste Management 40, 44-52.

Arellano, E., Ginocchio, R., 2013. Desafíos de las políticas públicas de gestión de residuos orgánicos en Chile para fomentar su reutilización en sistemas degradados. Centro de Políticas Públicas UC 64, 1 - 12.

Bernal, M.P., Clemente, R., Walker, D.J., 2007. The role of organic amendments in the bioremediation of heavy-metal polluted soils, in: Gore, R.B. (Ed.), Environmental research at the leading edge. Nova Publishing, New York. pp. 1-57.

Bernal, M.P., Clemente, R., Walker, D.J., 2009. Interactions of heavy metals with soil organic matter in relation to phytoremediation, in: Navarro-Aviño .JP. (Ed.), Phytoremediation: the green salvation of the world. Research Signpost, Trivandrum. pp. 109-129.

Cárcamo, V., Bustamante, E., Trangolao, E., de la Fuente, L.M., Mench, M., Neaman, A., Ginocchio, R., 2012. Simultaneous immobilization of metals and arsenic in acidic polluted soils near a copper smelter in central Chile. Environmental Science and Pollution Research. 19, 1131-
43. doi:10.1007/s11356-011-0673-3

Clemente, R., Walker, D., Pardo, T., Martínez-Fernández, D., Bernal, M., 2012. The use of a halophytic plant species and organic amendments for the remediation of a trace elements-contaminated soil under semi-arid conditions. Journal of Hazardous Materials 223-224, 63-71.

Conesa, H. M., Faz, A., Arnaldos, R., 2007. Initial studies for the phytostabilization of a mine tailing from the Cartagena-La Union Mining District (SE Spain). Chemosphere 66(1), 38-44. doi:10.1016/j.chemosphere.2006.05.041

De la Fuente, C., Clemente, R., Martínez, J., Bernal, P., 2010. Optimization of pig slurry application to heavy metal polluted soils monitoring nitrification processes. Chemosphere 81, 603-610.

De Varennes, A., Abreu, M.M., Qu, G., Cunha-Queda, C., 2010. Enzymatic activity of a mine soil varies according to vegetation cover and level of compost applied. International Journal of Phytoremediation 12, 371-383.

Espejo, C., García, R., 2009. Tratamiento de purines de ganado porcino en España para minimizar la contaminación de suelos y su impacto ambiental, en: Avances en estudios sobre desertificación: Aportaciones al Congreso Internacional sobre desertificación en memoria del Profesor John B. Thornes. Universidad de Murcia y la Sociedad Española de Geomorfología. pp. 673-676. http://congresos.um.es/icod/icod2009/paper/viewFile/5391/5181

Farrel, M., Griffith, G.W., Hobbs, P.J., Perkins, W.T., Jones, D.L., 2010. Microbial diversity and activity are increased by compost amendment on metal-contaminated soil. FEMS Microbiolgy Ecology 71, 94-105.

Giacomozzi, J., Amunátegui, R., 2016. Boletín carne bovina: tendencias de producción, precios y comercio exterior. http://www.odepa.cl/wp-content/files_mf/1456 428553Bolet\%C3\%ADndelacarne201602.pdf (acceso 25.03.2016)

Ginocchio, R., León-Lobos, P., 2010. Fitoestabilización de depósitos de relaves en Chile. Guía $\mathrm{N}^{\circ} 1$ : Metodología General, 1st ed. Centro de Investigación Minera y Metalúrgica, Santiago, Chile.

Hjorth, M., Christensen, K.V., Christensen, M.I., Sommer, S.G., 2010. Solid-liquid separation of animal slurry in theory and practice. A Review. Agronomy for Sustainable Development Dev. 30, 153-180.

Hooper, M., Anderson, M., 2009. Soil Toxicity and Bioassessment Test Methods for Ecological risk Assessment. Toxicity Test Methods for Soil Microorganisms, Terrestrial Plants, Terrestrial Invertebrates and Terrestrial Vertebrates. Environmental Protection Agency, California. https://oehha.ca.gov/media/downloads/ecotoxicology/document/soiltox120208.pdf

Hough, R. L., Tye, A. M., Crout, N. M. J., McGrath, S. P., Zhang, H., Young, S. D., 2005. Evaluating a 'Free Ion Activity Model' applied to metal uptake by Lolium perenne L. grown in contaminated soils. Plant and Soil 270(1), 1-12.

International Organization for Standarization (ISO), 1993. Soil quality - Determination of the effects of pollutants on soil flora Part 1: Method for the measurement of inhibition of root growth. https://law.resource.org/pub/in/ bis/S06/is.15109.1.2002.pdf (acceso 10.06.2016).

Kabas, S., Faz, A., Acosta, J. A., Zornoza, R., Martínez-Martínez, S., Carmona, D. M., Bech, J., 2012. Effect of marble waste 
and pig slurry on the growth of native vegetation and heavy metal mobility in a mine tailing pond. Journal of Geochemical Exploration 123, 69-76.

Kabas, S., Faz, A., Acosta, J.A., Arocena, J.M., Zornoza, R., Carmona, D.M., 2014. Marble wastes and pig slurry improve the environmental and plant relevant properties of mine tailings. Environmental Geochemistry and Health 41-54. doi:10.1007/s10653-013-9517-z

Kacprzak, M., Grobelak, A., Grosser, A., Prasad, M.N.V., 2014. Efficacy of biosolids in assisted phytostabilization of metalliferous acidic sandy soils with five grass species. International Journal of Phytoremediation 16(6), 593608. doi:10.1080/15226514.2013.798625.

Kumar, A., Maiti, S.K., 2015. Effect of Organic Manures on the Growth of Cymbopogon citratus and Chrysopogon zizanioides for the Phytoremediation of Chromite-Asbestos Mine Waste: A Pot Scale Experiment. International Journal of Phytoremediation 17(5), 437-447. doi:10.1080/1 5226514.2014 .910174

Li, X. F., Huang, L. B., 2015. Toward a New Paradigm for Tailings Phytostabilization-Nature of the Substrates, Amendment Options, and Anthropogenic Pedogenesis. Critical Reviews in Environmental Science and Techno$\operatorname{logy} 45,813-839$.

Lu, X.-M., Lu, P.-Z., Chen, J.-J., Zhang, H., Fu, J., 2015. Effect of passivator on $\mathrm{Cu}$ form transformation in pig manure aerobic composting and application in soil. Environmental Science and Pollution Research 22, 14727-14737.

Martínez-Fernández, D., Arco-Lazaro, E., Bernal, M. P., Clemente, R., 2014. Comparison of compost and humic fertiliser effects on growth and trace elements accumulation of native plant species in a mine soil phytorestoration experiment. Ecological Engineering 73, 588-597.

McLaughlin, R., Bartholomew, N., 2006. Soil Factors Influencing Suspended Sediment Flocculation by Polyacrylamide. Soil Science Society of America Journal 71 (2), 537-544.

Mendez, M.O., Maier, R.M., 2008. Phytostabilization of mine tailings in arid and semiarid environments. An emerging remediation technology. Environmental Health Perspectives 116(3), 278-283.

Ministerio del Medio Ambiente, 2010. Decreto 4: Reglamento para el manejo de lodos generados en plantas de tratamiento de aguas servidas. https://www.leychile.cl/ $\mathrm{N}$ ? $\mathrm{i}=1007456 \& \mathrm{f}=2010-04-26 \& \mathrm{p}$. (acceso 17.11.2016).

Pardo, T., Clemente, R., Bernal, M. P., 2011. Effects of compost, pig slurry and lime on trace element solubility and toxicity in two soils differently affected by mining activities. Chemosphere 84, 642-650.

Pardo, T., Clemente, R., Epelde, L., Garbisu, C., Bernal, M. P., 2014. Evaluation of the phytostabilisation efficiency in a trace elements contaminated soil using soil health indicators. Journal of Hazardous Materials 268, 68-76. doi:10.1016/j.jhazmat.2014.01.003

Peralta, M.J., Monreal, Z., Retamal, Y., Salazar, F., Ortega, F., Baeriswyl, F., Avedaño, L., 2005. Recomendaciones técnicas para la gestión ambiental en el manejo de purines de la explotación porcina. Instituto de Investigaciones Agropecuarias, Santiago, Chile. http://www2.inia.cl/ medios/catalogo/INIA_L0018.pdf. (acceso 23.06.2016)

Popovic, O., Jensen, L. S., 2012. Storage temperature affects distribution of carbon, VFA, ammonia, phosphorus, cop- per and zinc in raw pig slurry and its separated liquid fraction. Water Research 46, 3849-3858.

Santibáñez, C., Verdugo, C., Ginocchio, R., 2008. Phytostabilization of copper mine tailings with biosolids: Implications for metal uptake and productivity of Lolium perenne. Science of the Total Environment 395(1), 1-10. doi:10.1016/j.scitotenv.2007.12.033

Santibáñez, C., Ginocchio, R., Brown, S., 2011. Fitoestabilización de Depósitos de Relaves en Chile Guia N²: Aplicacion Sustentable de Acondicionadores. Centro de Investigación Minera y Metalúrgica e Instituto de Investigaciones Agropecuaria, Santiago.

Smith, R., Bradshaw, A. D., 1979. The use of metal tolerant plant populations for the reclamation of metalliferous wastes. Journal of Applied Ecology 16(2), 595-612.

Ssenku, J.E., Ntale, M., Origa, H.O., 2014. Physico-chemical characteristics of copper tailings and pyrite soils in Western Uganda : Implication for phytoremediation. International Journal of Environmental Monitoring and Analysis 2, 191-198. doi:10.11648/j.ijema.20140204.12

Tigini, V., Franchino, M., Bona, F., Varese, G. C., 2016. Is digestate safe? A study on its ecotoxicity and environmental risk on a pig manure. Science of The Total Environment 551-552, 127-132.

Ussiri, D., Lal, R., 2005. Carbon sequestration in reclaimed minesoils. Critical Reviews in Plant Sciences 24, 151-165. doi:10.1080/07352680591002147.

Vangronsveld, J., Cunningham, S., 1998. Introduction to concepts, in: Vangronsveld, J., Cunningham, S. (Eds.), In situ inactivation and phytorestoration of metal contaminated soils. Springer-Verlag and RG Landes Company, Berlin and Georgetown, pp. 1-15.

Vanotti, M. B., Hunt, P. G., 1999. Solids and nutrient removal from flushed swine manure using polyacrylamides. Transactions of the Asae 42, 1833-1840.

Verdugo, C., Sánchez, P., Santibáñez, C., Urrestarazu, P., Bustamante, E., Silva, Y., Gourdon, D., Ginocchio, R., 2011. Efficacy of lime, biosolids, and mycorrhiza for the phytostabilization of sulfidic copper tailings in Chile: a greenhouse experiment. International Journal of Phytoremediation $13,107-125$.

Ye, Z. H., Wong, J. W. C., Wong, M. H., Lan, C. Y., Baker, A. J. M., 1999. Lime and pig manure as ameliorants for revegetating lead/zinc mine tailings: a greenhouse study. Bioresource Technology 69, 35-43.

Zanuzzi, A., Arocena, J. M., Van Mourik, J. M., Cano, A. F., 2009. Amendments with organic and industrial wastes stimulate soil formation in mine tailings as revealed by micromorphology. Geoderma, 154, 69-75.

Zanuzzi, A., Faz, A., Acosta, J.A., 2013. Chemical stabilization of metals in the environment: a feasible alternative for remediation of mine soils. Environmental Earth Sciences 70, 2623-2632.

Zornoza, R., Faz, A., 2013. Marble waste and pig manure amendments decrease metal availability, increase soil quality and facilitate vegetation development in bare mine soils. Terra Latinoamericana 31, 105-114.

Zornoza, R., Faz, A., Carmona, D. M., Kabas, S., Martínez-Martínez, S., Acosta, J. A., 2012. Plant Cover and Soil Biochemical Properties in a Mine Tailing Pond Five Years After Application of Marble Wastes and Organic Amend- 
ments. Pedosphere 22, 22-32.

Zornoza, R., Faz, A., Carmona, D. M., Acosta, J. A., MartínezMartínez, S., De Vreng, A., 2013. Carbon mineralization, microbial activity and metal dynamics in tailing ponds amended with pig slurry and marble waste. Chemosphere 90, 2606-2613.

Zucconi, F., Pera, A., Forte, M., De Bertoldi, M., 1981. Evaluating toxicity of immature compost. BioCycle 22, 54-57. 